\section{P193 USEFUL I: MUSCULOSKELETAL ULTRASOUND TO IDENTIFY PATIENTS WITH LUPUS ARTHRITIS WITH BETTER RESPONSE TO THERAPY}

\begin{abstract}
1,2Khaled Mahmoud, ${ }^{3}$ Ahmed S Zayat, ${ }^{1,2}$ Md Yuzaiful Md Yusof, ${ }^{1,2}$ Katherine Dutton, ${ }^{4}$ Lee Suan Teh, ${ }^{5}$ Chee-Seng Yee, ${ }^{6}$ David D'Cruz, ${ }^{6}$ Nora Ng, ${ }^{7}$ David Isenberg, ${ }^{7}$ Coziana Ciurtin, ${ }^{1,2}$ Philip Conaghan, ${ }^{1,2}$ Paul Emery, ${ }^{8}$ Chris Edwards, ${ }^{1,2}$ Elizabeth MA Hensor, ${ }^{1,2}$ Edward M Vital. 'NIHR Leeds Biomedical Research Centre, Leeds Teaching Hospitals NHS Trust, Leeds; ${ }^{2}$ Leeds Institute of Rheumatic and Musculoskeletal Medicine, University of Leeds, Leeds, UK; ${ }^{3}$ Bradford Teaching Hospitals NHS Foundation Trust, Bradford; ${ }^{4}$ Royal Blackburn Teaching Hospital, Blackburn and University of Central Lancashire, Preston; ${ }^{5}$ Doncaster and Bassetlaw NHS Trust, Doncaster; ${ }^{6}$ Guys and St Thomas Hospital, London; ' University College London, London; ${ }^{8}$ University Hospital Southampton NHS Foundation Trust, Southampton
\end{abstract}

10.1136/lupus-2020-eurolupus.235

Background In SLE, musculoskeletal manifestations have an impact on quality of life, disability and clinical trial outcomes, but are harder to assess than in RA and PsA. We previously showed that joint swelling lacks sensitivity, specificity and responsiveness compared to ultrasound. USEFUL was a multicentre longitudinal study to determine clinical features predicting ultrasound synovitis and whether patients with ultrasound synovitis respond better to therapy.

Methods SLE patients were recruited if the referring physician deemed they had inflammatory pain warranting treatment. Swollen joints were not required. At baseline, physicians recorded the features that led them to diagnose inflammatory pain and features of concurrent fibromyalgia and osteoarthritis. Stable doses of prednisolone $(\leq 5 \mathrm{mg} /$ day $)$, antimalarials or immunosuppressants were allowed. Participants received depomedrone $120 \mathrm{mg}$ IM then were assessed at 0,2 and 6 weeks for 66/68 swollen and tender joint counts, BILAG-2004, SLEDAI-2K, physician global and MSK-VAS, inflammatory markers, patient pain and disease activity-VAS, HAQ-DI, LupusQoL, ultrasound of hands and wrists (blinded to patient and clinical assessor). An internal pilot determined the primary endpoint: EMS-VAS at 2 weeks (adjusted for baseline) between patients with ultrasound-synovitis vs. normal ultrasound at baseline. Sensitivity analyses adjusted for prednisolone and immunosuppressants.

Results 122/133 patients recruited completed all visits. There was significant disagreement between clinical examination and ultrasound. $78 / 133$ had ultrasound synovitis; $68 \%$ of these had $\geq 1$ swollen joint. Of $66 / 133$ patients with $\geq 1$ swollen joint, 20\% had normal ultrasound.

Ultrasound-synovitis was more likely with joint swelling, a symmetrical small joint distribution and active serology. Physician-determined EMS, other lupus features or prior response to therapy were not associated. Fibromyalgia or osteoarthritis did not reduce the probability of ultrasound synovitis.

In the full analysis set $(n=133)$ there was no difference in EMS VAS at 2 weeks according to ultrasound synovial status as baseline (difference $-8 \mathrm{~mm}, 95 \%$ CI $-19,4 \mathrm{~mm}, \mathrm{p}=0.178$ ). 32 patients had fibromyalgia. After excluding these patients, we found a statistically and clinically significantly better clinical response to depomedrone in patients with ultrasound-synovitis at baseline (baseline-adjusted EMS VAS at 2 weeks $-12 \mathrm{~mm}$, 95\% CI $-24,0 \mathrm{~mm}, \mathrm{p}=0.049)$. This difference was greater in the treatment-adjusted sensitivity analysis $(-12.8$ (95\% CI $-22,-3$ $\mathrm{mm}), \mathrm{p}=0.007)$ and the per-protocol-adjusted sensitivity analysis $(-14.8 \mathrm{~mm}$ (95\% CI $-20.8,-8.8 \mathrm{~mm}), \mathrm{p}<0.001)$. Patient with ultrasound synovitis had higher rates of improvement in the musculoskeletal BILAG-2004 (56\% vs. 26\%, $\mathrm{p}=0.09)$ and SLEDAI-2K (37\% vs. $15 \%, \mathrm{p}=0.03)$.

Conclusions In lupus arthritis distribution and serology, but not other features, help identify ultrasound-synovitis. Ultrasound-synovitis was independent of features of fibromyalgia, but fibromyalgia confounded assessment of response. Excluding fibromyalgia, response to therapy was better in patients with abnormal ultrasound compared to normal. Ultrasound should be used to select patients for therapy and clinical trials, especially when there are inflammatory symptoms without swollen joints.

Acknowledgements This project was funded by a grant from Lupus UK. 